\title{
First Undulators For The Advanced Light Source
}

\author{
E. Hoyer, J. Akre, J. Chin, B. Gath, D. Humphries, B. Kincaid, S. Marks, P. Pipersky, D. Plate, G. Portmann, and R. Schlueter \\ Lawrence Berkeley Laboratory, University of California, Berkeley, California 94720
}

\author{
W. V. Hassenzahl
}

Lawrence Livermore National Laboratory, University of California, Livermore, California 94551

\section{Abstract}

The first three undulators, each $4.6 \mathrm{~m}$ in length, for the Advanced Light Source (ALS) at Lawrence Berkeley Laboratory (LBL), are near completion and are undergoing qualification tests before installation into the storage ring. Two devices have $5.0-\mathrm{cm}$ period lengths, 89 periods, and achieve an effective field of $0.85 \mathrm{~T}$ at the $14 \mathrm{~mm}$ minimum magnetic gap. The other device has a period length of $8.0 \mathrm{~cm}$, 55 periods, and an effective field of $1.2 \mathrm{~T}$ at the minimum $14 \mathrm{~mm}$ gap. Measurements on the first $5 \mathrm{~cm}$ period device show the uncorrelated field errors to be $0.23 \%$, which is less than the required $0.25 \%$. Measurements of gap control show reproducibility of \pm 5 microns or better. The first vacuum chamber, $5.0 \mathrm{~m}$ long, is flat to within $0.53 \mathrm{~mm}$ over the $4.6 \mathrm{~m}$ magnetic structure section and a $4 \times 10^{-11}$ Torr pressure was achieved during vacuum tests. Device description, fabrication, and measurements are presented.

\section{INTRODUCTION}

The ALS, a third-generation synchrotron radiation source is currently being commissioned at I.BL. Concurrently, the first ALS undulators are nearing completion, undergoing qualification testing, and are to be installed soon in the lowemittance $1.5 \mathrm{GeV}$ storage ring to produce high brightness beams in the UV to soft $x$-ray range. Two devices have $5.0-\mathrm{cm}$ period lengths (IDA-U5.0 and IDB-U5.0), and the third device has an $8.0-\mathrm{cm}$ period length (IDC-U8.0), which will produce high brightness in the 50 to $1500 \mathrm{eV}$ and 6 to $1000 \mathrm{eV}$ ranges respectively. The undulator specifications and design have been reported elsewhere $[1,2$, and 3$]$; the first U5.0 Undulator near completion is shown in Figure 1.

\section{MAGNETIC STRUCTURE}

As seen in Figure 1, the magnetic structure includes two large backing beams, each has five assembly sections and two end pole structures attached. Six low-reluctance flux shunts connect the two backing beams magnetically. A U5.0 assembly section, Figure 2, consists of 35 half-period pole assemblies, each bolted to a pole mount.

The basic building block of the magnetic structure is the half period pole assembly which consists of an aluminum keeper, a Vanadium Permendur pole and six Nd-Fe-B magnetized blocks. Manufacture of these assemblies is as follows:

- Nd-Fe-B blocks are ordered with stringent specifications

which include $\mathrm{H}_{\mathrm{C}} \geq 10400$ Oe [4].
- Blocks are inspected for defects, mechanical dimensions, magnetic imperfections and measured using an automated Helmholtz coil system [5]. Results show that the 4,582 U5.0 blocks have average casy axis magnetization of $1.1137 \mathrm{~T}(\sigma=0.0110 \mathrm{~T})$ and average minor axis magnetization components of $0.0031 \mathrm{~T}(\sigma=0.0044 \mathrm{~T})$ and $0.0011 \mathrm{~T}(\sigma=0.0043 \mathrm{~T})[6]$.

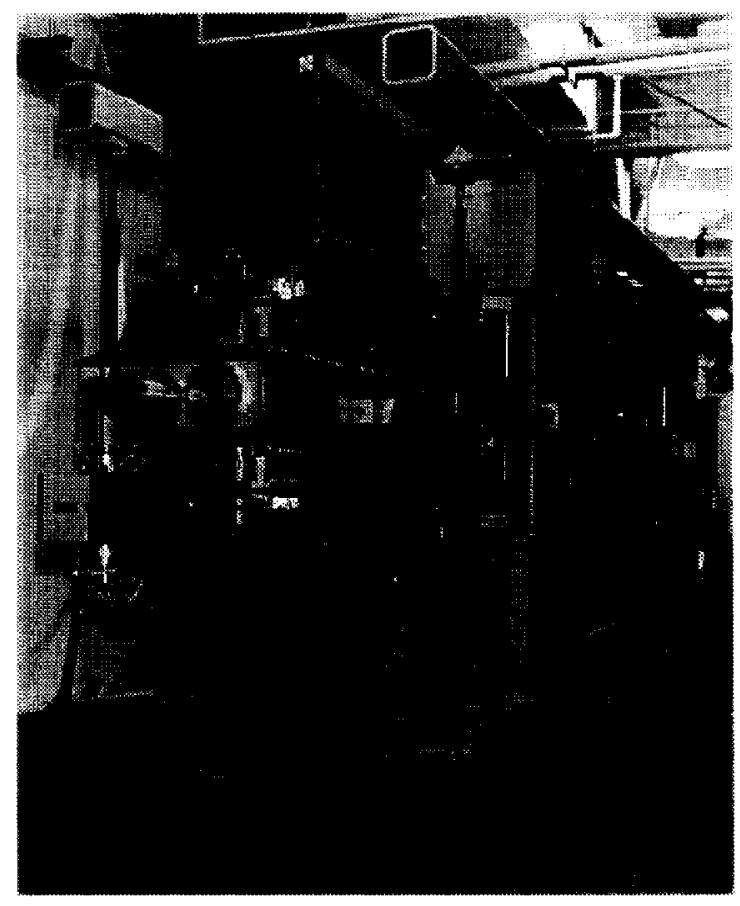

Figure 1. U5.0 Undulator

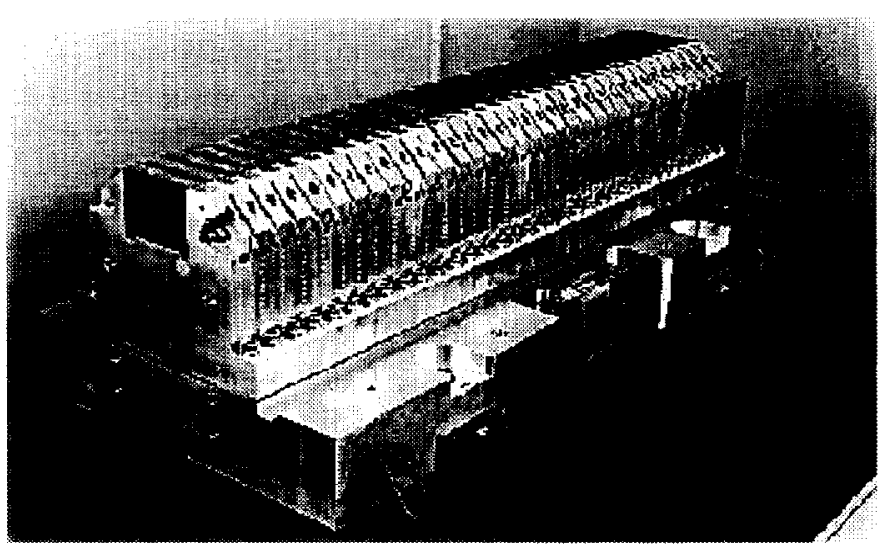

Figure 2. U5.0 Undulator Assembly Section 
- Blocks are sorted into the six-block arrays. The sorting algorithm, code INSORT, minimizes pole excitation errors, reduces direct field effects at the electron beam, and generates assembly diagrams showing where each block goes in each half-period pole assembly.

- The Vanadium Permendur poles are machined, heat treated in a hydrogen furnace at 875 degrees $C$ for four hours and then finish machined. Each pole is pinned to its keeper with four stainless pins.

- The Nd-Fe-B blocks are positioned with a bonding fixture and bonded into the pole-keeper assembly with a room temperature curing epoxy (Technicol 8260/8261) [7].

Assembly section fabrication utilizes a milling machine and a pole assembly holding fixture to place and bolt the half-period pole assemblies to the pole mount. To achieve the $25 \mu \mathrm{m}$ vertical and $50 \mu \mathrm{m}$ longitudinal pole tip positional tolerances, the half-period pole assemblies are aligned using an automated coordinate measurement machine, statistically-based error reference planes, and shimming techniques.

The aligned assembly sections are installed on the backing beams and aligned relative to each other using optical tooling and laser interferometric techniques [8]. The longitudinal positions of the assembly sections are measured using a simple linear interferometer and associated optics. Because of the differential expansion coefficient between the aluminum assembly section and the steel backing beam, a bi-linear temperature compensation function is applied to the position data to predict periodicity errors at a pre-determined operating temperature which in general differs from measurement temperatures.

The vertical alignment of the assembly section reference planes, to within a $12 \mu \mathrm{m}$ tolerance, is obtained by using an angular interferometer, and performing an integrated angle calculation on the data. Repeatability error for these measurements is typically less than $3 \mu \mathrm{m}$.

The magnetic structure is terminated with end structures that contain $\mathrm{Nd}-\mathrm{Fe}-\mathrm{B}$ rotor assemblies to null the undulator dipole field. These rotors are driven through linkages from the backing beams and can be positioned to within 2 degree $[9$ and 10 ].

Each end structure is outfitted with a multiple trim magnet cartridge that corrects higher order magnetic field errors in the undulator. A cartridge contains up to nine transversely located permanent magnets (Nd-Fe-B) that are vertically oriented and adjustable to correct for both vertical and horizontal undulator field integral errors [11].

The backing beams are of low carbon steel construction and stress-relieved at 600-700 degrees $\mathrm{C}$ for four hours. The $\mathrm{Ni}-\mathrm{Fe}$ flux shunts connecting the upper and lower backing beams reduce the effect of environmental magnetic fields on the undulator magnetic field [12].

\section{SUPPORT/DRIVE /CONTROL SYSTEMS}

A generic support/drive/control system is used for the ALS undulators. The support and drive systems, shown in
Figure 1, include the support structure that provides the framework for holding the magnetic structure and the drive system that opens and closes the magnetic gap. Gap motion is achieved with a stepper-motor/gear box/roller chain drive with coupled left-hand \& right-hand $2 \mathrm{~mm}$ pitch Transrol roller screws attached to the upper and lower backing beams [13]. Adjustment of the magnetic gap can be as fine as $5 \mu \mathrm{m}$ through the use of offset keys in the roller screw shaft couplings. Compensating springs match the gap dependent magnetic load to within $20 \%$. Magnetic field taper can be provided manually by stepping one of the main roller chain sprocket's teeth with respect to the chain in increments of $87 \mu \mathrm{m} /$ sprocket tooth $(\Delta \mathrm{E} / \mathrm{E}=0.96 \%$ at $1.4 \mathrm{~cm}$ gap $)$.

A Compumotor system, Indexer Model No. 500-Driver Model PK130M-Absolute Rotary Encoder Model ARC, is used to drive and control the magnetic gap. This stepper motor and absolute rotary encoder system is designed such that each motor step and encoder step corresponds to $0.067 \mu \mathrm{m}$ and $0.106 \mu \mathrm{m}$ motion of the gap respectively. The motor control system also allows for velocity profiling during a move. Results of support, drive, and control system tests are given in Table 1.

Table 1.

Support/drive/control system test results

\begin{tabular}{|ll|}
\hline Test & Performance \\
\hline \hline Magnetic structure & Gap parallelism within $5 \mu \mathrm{m} ;$ \\
alignment (upper-to-lower & transverse within $250 \mu \mathrm{m} ;$ \\
structure) & longitudinal within $150 \mu \mathrm{m}$ \\
Gap range & $14 \mathrm{~mm}$ to $210 \mathrm{~mm}$ \\
Gap opening-closing time & 1 minute \\
Reproducibility & $\leq \pm 5 \mu \mathrm{m} ; \pm 20 \%$ for $1 \mu \mathrm{m}$ \\
& steps \\
Backlash (encoder to gap) & $\leq 10 \mu \mathrm{m}$ \\
Scan rate & $3.33 \mathrm{~mm} / \mathrm{sec}$ max. \\
\hline
\end{tabular}

A gage block and a laser interferometer is used to calibrate the absolute encoder to the gap position. Figure 3 shows the deviation from linearity with and without compensating springs (three hysteresis loops each). The hacklash of the system has gap dependence, and the character of the upper hysteresis curve is due to the compensating springs not exactly canceling the force of the magnetic field. Nonlinearities are removed with calibration.

Each undulator is outfitted with a transparent enclosure for safety, and for maintaining uniform temperature throughout the insertion device. Tests show that the gap sensitivity coefficient, due to a vertical temperature gradient in a backing beam, is $6 \mu \mathrm{m} / 0.1$ degree $C$ and that the temperature gradient is eliminated when fans circulate air within the enclosure.

\section{VACUUM SYSTEM}

The $5.1 \mathrm{~m}$ long IDA-U5.0 commissioning vacuum chamber, shown in Figure 1, is machined from two 5083-H321 aluminum plates and welded together [14]. Pockets machined 
into the chamber accommodate the poles of the magnetic structure for small magnetic gap operation. Mechanical measurements taken at the completion of final assembly, after UHV conditioning and under vacuum, are given in Table 2.

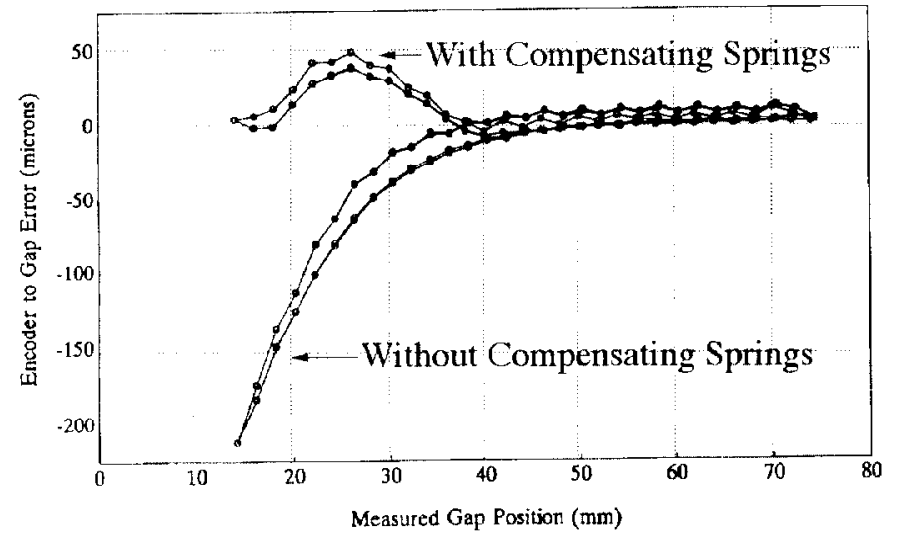

Figure 3. Hysteresis Loops with and without compensating springs for IDB-U5.0.

Table 2.

ALS Commissioning Vacuum Chambers Measurements

\begin{tabular}{|lcrl|}
\hline Measurement & IDA-U5.0 & IDB-U5.0 & IDC-U8.0 \\
\hline \hline Vertical aperture & $18.97 \mathrm{~mm}$ & $18.83 \mathrm{~mm}$ & In progress \\
Min. v.c. gap & $21.64 \mathrm{~mm}$ & $21.77 \mathrm{~mm}$ & In progress \\
V.C. flatness & $0.53 \mathrm{~mm}$ & $0.74 \mathrm{~mm}$ & In progress \\
Hor. straightness & $0.08 \mathrm{~mm}$ & $0.05 \mathrm{~mm}$ & In progress \\
\hline
\end{tabular}

The pumping system of each vacuum chamber includes three combination $60 \mathrm{l} / \mathrm{s}$ ion- $600 \mathrm{l} / \mathrm{s}$ titanium sublimation pumps, a $2400 \mathrm{l} / \mathrm{s}$ ALS absorber tsp pump, and $4800 \mathrm{l} / \mathrm{s}$ of non-evaporable getter (Saes-type ST707/CTAM/30D strip).

Chamber ultra-high vacuum conditioning is at $140 \mathrm{de}$ grees $\mathrm{C}$, for a minimum of 48 hours [15]. The base pressure achieved after conditioning is $3.7 \times 10^{-11}$ Torr for the IDAU5.0 chamber and $7.0 \times 10^{-11}$ Torr for the IDB-U5.0 chamber.

\section{MAGNETIC MEASUREMENTS}

A high speed, precision magnetic measurement system has been designed and built for detailed magnetic characterization of ALS insertion devices [16]. Primary magnetic field information, integral and local data, are derived from Hall probe measurements. A $5.5 \mathrm{~m}$ long integral coil is also used to measure field integrals.

- The rms harmonic residual, defined as the rms value of the deviation of the measured field on axis from the ideal field, provides an indication of spectral performance [17]. For IDA - U5.0, the specified rms harmonic residual is $0.25 \%$; magnetic measurcments show a value of $0.23 \%$.

Field integrals through IDA are close to specified tolerances and very acceptable for ALS operations.

\section{ACKNOWLEDGMENT}

*This work was supported by the Director, Office of Energy Research, Office of Basic Energy Sciences, Materials Sciences Division, of the U. S. Department of Energy, under Contract No. DE-AC03-76SF00098.

\section{REFERENCES}

[1] "U5.0 Undulator Conceptual Design Report," LBL PUB-5256 (Nov. 1989).

[2] "U8.0 Undulator Conceptual Design Report," LBL PUB-5276 (May 1990).

[3] E. Hoyer, et. al., "The U5.0 Undulator for the ALS, 4th International Conf. on Sync. Rad. Inst.," [LBL-30459], Rev., Sci. Instrum. 63 (1), 359 (January 1992).

[4] E. Hoyer, "U5.0 Magnetic Structure Magnetized Nd-FeB Blocks," LBL Spec. M734D (Apr. 1989)

[5] S. Marks, et. al., "ALS Insertion Device Block Measurement and Inspection," [LBL-29955], IEEE PAC. 91CH3038-7, 2739 (May 1991).

[6] S. Marks, "Measurement \& Characterization of U5.0 Permanent Magnet Blocks," LBL Eng. Note M7225 (Oct. 1991).

[7] D. Humphries, "Block Bonding Procedure for IDA/IDB/IDC," LBL Eng. Note M7121 (Feb. 1991)

[8] B. Gath, "Magnetic Structure Installation and Alignment into Backing Beam," LBL Eng. Note M7265 (Mar. 1992).

[9] D. Humphries, et al, "Modeling and Measurement of the ALS U5.0 Undulator End Magnetic Structure," IEEE PAC (May 1993).

[10] P. Pipersky, "U5.0 End Module Assembly \& Installation," LBL Eng. Note M7277A (April 1992).

[11] E. Hoyer, "Multiple Trim Magnets," LBL Eng. Note M7354 (May 1993).

[12] E. Hoyer, et. al, "Flux Shunts for Undulators,".IEEE PAC (May 1993).

[13] J. Chin, "IDA, IDB, IDC Support \& Drive System Assembly and Alignment Notes," LBL Eng. Note M7349 (July 1992).

[14] D. Plate, "Fabrication Procedure \& Production Inspection Record for Vacuum Chambers," LBL Eng. Note M7292A (Aug. 1992).

[15] D. Plate, "Insertion Device Vacuum Chamber Bakeout Procedure,” LBL Eng. Note M7304 (Sept. 92).

[16] S. Marks, et al, "ALS Insertion Device Magnetic Measurements," IEEE PAC (May 1993).

[17] C. Wang, et. al, "Calculated Radiation Spectrum of ALS U5.0 Undulator Based Upon Magnetic Measurements," IEEE PAC (May 1993). 